\title{
CONSTRUCTIVE SOLUTION OF THE 1918 PROBLEM OF LORD RAYLEIGH
}

\author{
M. N. MCALLISTER, S. M. ROHDE AND G. T. MCALLISTER ${ }^{1}$
}

\begin{abstract}
In 1918 Lord Rayleigh posed the problem of finding the profile (shape) of a one dimensional slider bearing which will support the greatest weight. In this paper we give, in closed form, the solution of this problem.
\end{abstract}

1. Introduction. The pressure distribution for a one dimensional slider bearing with profile $H(x)$ is given as a solution of the Reynolds equation

$$
\left(H^{3}(x) p^{\prime}(x)\right)^{\prime}-H^{\prime}(x)=0
$$

with $p(0)=p(a)=0$. Here we are assuming that the slider is moving with unit speed and that the minimum film thickness is one (i.e., $H(x) \geqslant 1$ ). The weight that the slider bearing will support is given by the functional

$$
\mathcal{L}(p)=\int_{\{x: p(x)>0\}} p(x) d x .
$$

In 1918 Rayleigh showed surprisingly in [1] that there exists a profile with a simple jump discontinuity which supports more weight than do profiles of the form $1+m x^{n}$ or $e^{\beta x}$ with $m, n$ and $\beta$ positive. He also posed the problem of finding a profile which will support the greatest weight; in this regard he only found a profile which caused the first variation of the weight functional to vanish. It is the object of this paper to mathematically solve, in closed form, this unresolved problem.

We will solve the problem of Rayleigh over the set of admissible bearing profiles $\mathcal{H}=\left\{H: H \in L_{\infty}((0, a)), M \geqslant H(x) \geqslant 1\right.$ a.e. over $\left.(0, a)\right\}$ with $M>1$ and $a \in(0, \infty)$ the given physical parameters. With this generality in $\mathcal{H}$ we must reformulate (1). For each $H(x) \in \mathcal{H}$ we let $p_{H}(x) \in H_{2,0}^{1}((0, a))$ be such that

$$
\int_{0}^{a}\left\{H^{3}(x) p_{H}^{\prime}(x) \zeta^{\prime}(x)-H(x) \zeta^{\prime}(x)\right\} d x=0
$$

for all $\zeta(x) \in H_{2,0}^{1}((0, a))$; for a detailed description of this function space see Morrey [2]. Such a function $p_{H}(x)$ is the pressure distribution under the slider bearing and the subscript $H$ is used to denote its dependence on the specific profile $H$. We now have the problem of Rayleigh: Find an $H_{0} \in \mathcal{H}$ such that

$$
\mathcal{L}\left(p_{H_{0}}\right)=\max \left\{\mathcal{L}\left(p_{H}\right): H \in \mathcal{H}\right\}
$$

Received by the editors May 13, 1978.

AMS (MOS) subject classifications (1970). Primary 49A99; Secondary 49A25.

${ }^{1}$ Research partially supported by NSF Grant MCS 77-05624. 
where the functional $\mathcal{L}(\cdot)$ is defined in (2). Note that our $\mathcal{H}$ contains functions with any number of discontinuities.

The functional in (2) is difficult to evaluate for $H \in \mathcal{H}$ as we must know the region of nonnegativity of $p_{H}(x)$ before we can even integrate. Consequently we will also solve the problem in (3) when $\mathcal{L}(\cdot)$ is replaced by the functional

$$
\text { थ }\left(p_{H}\right)=\int_{0}^{a} p_{H}(x) d x .
$$

The problem we have outlined here is sometimes called a distributed parameter problem; the control variable $H$ occurs in the coefficient of the highest order derivative. Our solution of (3), or (3) with (4), represents a very rare case that these problems have a closed form solution.

In $\$ 2$ we construct the profile $H_{0}$ which solves (3) and the problem in (3) with $\mathcal{L}(\cdot)$ replaced by $\mathscr{W}(\cdot)$. This $H_{0}$ is a step function with one jump discontinuity and $\mathcal{L}\left(p_{H_{0}}\right)=\mathscr{W}\left(p_{H_{0}}\right)$; i.e. the problem of Rayleigh has the same solution whether the functional in (2) or (4) is used.

The condition that the slider is moving at unit speed is imposed only for algebraic convenience. Our methods extend trivially to the case of arbitrary speed.

In the sequel $\|f\|_{2}^{2}=\int_{0}^{a}(f(x))^{2} d x$.

2. Constructive solution of the problem of Rayleigh. We begin with a simple result.

LEMMA 2.1. For each $H \in \mathcal{K}$ there exists a unique $p_{H}(x) \in H_{2,0}^{1}((0, a))$ such that

$$
\int_{0}^{a}\left\{H^{3}(x) p_{H}^{\prime}(x) \zeta^{\prime}(x)-H(x) \zeta^{\prime}(x)\right\} d x=0
$$

for every $\zeta(x) \in H_{2,0}^{1}((0, a))$. Moreover,

$$
\left\|p_{H}^{\prime}(x)\right\|_{2} \leqslant\|H\|_{2} \leqslant M a^{1 / 2}
$$

and, consequently, $p_{H}(x)$ is Hölder continuous with constant $M a^{1 / 2}$ and exponenent $\frac{1}{2}$.

The next lemma follows from [3, Lemma 1.1, p. 67].

LeMma 2.2. For each $H \in \mathcal{H}$ there exists a constant $C(H)$, which depends on $H$, such that

$$
H^{3}(x) p_{H}^{\prime}(x)-H(x)=C(H)
$$

for almost all $x \in[0, a]$. Moreover

$$
C(H)=-\int_{0}^{a} \frac{d x}{H^{2}} / \int_{0}^{a} \frac{d x}{H^{3}} .
$$

Now we determine the range of the function $C(H)$ acting on $\mathcal{H}$. 
LEMMA 2.3. For each $H \in \mathcal{H},-C(H) \in[1, M]$ with $-C(H)=1$ $(-C(H)=M)$ if and only if $H(x)=1(H(x)=M)$ a.e. on $[0, a]$.

Proof. If $H \in \mathcal{H}, H^{3}(x) \geqslant H^{2}(x)$ and

$$
\int_{0}^{a} \frac{d x}{H^{3}(x)} \leqslant \int_{0}^{a} \frac{d x}{H^{2}(x)} .
$$

Therefore $-C(H) \geqslant 1$ with equality if and only if $H(x)=1$ a.e. Since $M \geqslant H(x), M H^{2}(x) \geqslant H^{3}(x)$ and

$$
\int_{0}^{a} \frac{d x}{H^{2}(x)} \leqslant M \int_{0}^{a} \frac{d x}{H^{3}(x)}
$$

with equality if and only if $H(x)=M$ a.e. The result follows from (2.4).

Now we improve our estimate in (2.2).

Lemma 2.4. For each $H \in \mathcal{H}$ let $F(x)=(H(x)+C(H)) / H^{3}(x)$ with $x \in$ $(0, a)$. Let

$$
A_{1}=\operatorname{ess} \sup \{F(x): x \in(0, a)\} \quad \text { and } A_{2}=\operatorname{ess} \inf \{F(x): x \in(0, a)\} \text {. }
$$

Then (i) $A_{1} \cdot A_{2} \leqslant 0$ with equality if and only if $H(x)$ is equivalent to a constant function on $(0, a)$, and (ii)

$$
A_{2} \leqslant p_{H}^{\prime}(x) \leqslant A_{1} \text { a.e. }
$$

Proof. It is clear that (2.5) follows from (2.3).

If $A_{1} \cdot A_{2}>0$ then we contradict $p_{H}(a)=0$ as $p_{H}(x)=\int_{0}^{x} p_{H}^{\prime}(x) d x$ remains valid for $p_{H} \in H_{2,0}^{1}((0, a))$. Therefore $A_{1} \cdot A_{2} \leqslant 0$.

Suppose $A_{1} \cdot A_{2}=0$. Let $A_{1}=0$. Then $A_{2}=0$, else we cannot satisfy the boundary condition $p_{H}(a)=0$. Hence $H$ is essentially equivalent to a constant function. Clearly, if $H$ is constant, $A_{1}=A_{2}=0$.

The next result shows that $\mathcal{H}$ may be decomposed into subsets.

LEMMA 2.5. For each $c \in[-M,-1]$, let

$$
\mathcal{H}_{c}=\{H: H \in \mathcal{K}, C(H)=c\} \text {. }
$$

Then

$$
\mathcal{H}=\bigcup\left\{\mathcal{H}_{c}: c \in[-M,-1]\right\}
$$

Proof. Clearly $\cup\left\{\mathcal{H}_{c}: c \in[-M,-1]\right\} \subset \mathcal{H}$. Let $H \in \mathcal{H}$. By Lemma 2.3 there exists $c \in[-M,-1]$ such that $C(H)=c$; i.e., $H \in \mathcal{H}_{c}$.

Let $c$ be any element of $[-1,-M]$. Consider the function $G_{c}(X)=(X+$ c) $/ X^{3}$ with $X \in[1, M]$. Since $G_{c}^{\prime}(X)=0$ implies that $X=-3 c / 2$ with $G_{c}^{\prime \prime}(-3 c / 2)<0$ and

$$
G_{c}^{\prime}(X)=-(2 X+3 c) / X^{3}>0
$$


for $-3 c>2 M$, then

$$
\begin{aligned}
\max \left\{G_{c}(X): X \in[1, M]\right\} & \\
= & \begin{cases}4 / 27 c^{2} & \text { for }-2 M / 3<c<-1 \text { and } M \geqslant 3 / 2, \\
(M+c) / M^{3} & \text { for }-M<c<-2 M / 3 \text { or } M<3 / 2 ;\end{cases}
\end{aligned}
$$

here $G_{c}(M)=(M+c) / M^{3}$ and $G_{c}(1)=1+c \leqslant 0$. The condition that $M$ $\geqslant 3 / 2$ above assures us that $-2 M / 3 \leqslant-1$. It is clear that $G_{c}(M)-$ $G_{c}\left(4 / 27 c^{2}\right) \leqslant 0(\geqslant 0)$ for $c \in[-2 M / 3,-1]$ (for $\left.c \in[-M,-2 M / 3]\right)$.

Also

$$
\min \left\{G_{c}(X): X \in[1, M]\right\}=1+c .
$$

For each $c \in[-2 M / 3,-1]$ and $M \geqslant 3 / 2$ we define the piecewise linear function

$$
l_{c}(x)= \begin{cases}4 x / 47 c^{2} & \text { for } x \in[0, \alpha], \\ (1+c)(x-a) & \text { for } x \in[\alpha, a],\end{cases}
$$

with $\alpha=-27 c^{2} a(1+c) /\left(4-27 c^{2}(1+c)\right)$ and for each $c \in$ $[-M,-2 M / 3)$ or $M<3 / 2$ we set

$$
l_{c}(x)= \begin{cases}(M+c) x / M^{3} & \text { for } x \in[0, \alpha], \\ (1+c)(x-a) & \text { for } x \in[\alpha, a],\end{cases}
$$

with $\alpha=-M^{3}(1+c) a /\left(M+c-(1+c) M^{3}\right)$.

Clearly the $\alpha$ associated with $(2.9)$ or $(2.10)$ is in $[0, a]$ for any $c \in$ $[-M,-1]$.

The next result gives bounds on $p_{H}(x)$ and its functionals.

LEMMA 2.6. Let $c$ be a fixed element of $[-M,-1]$, let $H$ be any element of $\mathcal{H}_{c}$, and let $p_{H}$ be the solution to (2.1) corresponding to this $H$. Then, for each $x \in[0, a], p_{H}(x) \leqslant l_{c}(x)$ and $l_{c}(x) \geqslant 0$; depending on the size of $c$ or $M$ we use (2.9) or (2.10). Therefore, for any $H \in \mathcal{H}_{c}$,

$$
\int_{I_{H}} p_{H}(x) d x \leqslant \int_{0}^{a} l_{c}(x) d x
$$

and

$$
\int_{0}^{a} p_{H}(x) d x<\int_{0}^{a} l_{c}(x) d x=\frac{a}{2} l_{c}(\alpha)
$$

where $I_{H}=\left\{x: x \in(0, a), p_{H}(x) \geqslant 0\right\}$.

PRoof. If $x \in[0, \alpha]$, then $p_{H}^{\prime}(x) \leqslant l_{c}^{\prime}(x)$ a.e. implies that $p_{H}(x) \leqslant l_{c}(x)$. Also, for $x \in(\alpha, a]$,

$$
p_{H}(x)=-\int_{x}^{a} p_{H}^{\prime}(\xi) d \xi \leqslant-\int_{x}^{a}(1+c) d \xi=(1+c)(x-a) .
$$


Hence $p_{H}(x)<l_{c}(x)$ in $[0, a]$. From this the bounds in (2.11) easily follow.

We will establish sufficient conditions that there exists an $H_{c} \in \mathcal{H}_{c}$ such that $l_{c}(x)$, as given in (2.9) or (2.10), satisfies (2.1) with $H=H_{c}$.

Since $l_{c}(x)$ is piecewise linear, that problem is equivalent to finding constants $A, B \in[1, M]$ such that either

$$
4 A^{3} / 27 c^{2}-A-c=0 \text { or }(M+c) A^{3} / M^{3}-A-c=0
$$

and

$$
(1+c) B^{3}-B-c=0 .
$$

If such $A, B \in[1, M]$ exist, then $H_{c}(x)=A$ for $x \in[0, \alpha)$ and $H_{c}(x)=B$ for $x \in[\alpha, a]$ would be in $\mathcal{H}_{c}$ and $l_{c}(x)$ would satisfy (2.1) with $H=H_{c}$.

For the first equation in (2.12), there is only one positive root given by $A=-3 c / 2$ and in the second equation there is only the real $\operatorname{root} A=M$.

In (2.13) we may always take $B=1$.

Let $c \in[-M,-1]$ be given. Then $l_{c}(x)$ solves (2.3) with $H=H_{c}$ as described above. From (2.11) we deduce that

$$
\max \left\{\int_{0}^{a} p_{H}(x) d x: H \in \mathscr{K}_{c}\right\}=\frac{a}{2} l_{c}(\alpha)=\max \left\{\int_{I_{H}} p_{H}(x) d x: H \in \mathscr{H}_{c}\right\} .
$$

Moreover, these maxima are assumed by the $H_{c}$ as described above. Therefore to solve the problem of Rayleigh we must obtain the value of $c$ for which $a l_{c}(\alpha) / 2$ is the largest possible.

Now we will determine the values of $c \in[-M,-1]$ so that the triangle with vertices $(0,0),(a, 0)$, and $\left(\alpha, l_{c}(\alpha)\right)$ has the largest area; i.e. find $c_{0}$ so that

$$
\frac{1}{2} a l_{c_{0}}(\alpha)=\max \left\{\frac{1}{2} a l_{c}(\alpha): c \in[-M,-1]\right\} .
$$

Setting $Q(c)=\frac{1}{2} a l_{c}(\alpha)$ we have

$$
\mathscr{Q}(c)=\left\{\begin{array}{c}
-2 a^{2}(1+c) /\left(4-27 c^{2}(1+c)\right), \\
-2 M / 3 \leqslant c \leqslant-1 \text { and } M>3 / 2, \\
-a^{2}(M+c)(1+c) / 2\left(M+c-(1+c) M^{3}\right), \\
-M \leqslant c<-2 M / 3 \text { or } M<3 / 2 .
\end{array}\right.
$$

Note that $\mathscr{Q}(c)$ is given by (2.15) whenever $M<3 / 2$. In both of these expressions $\mathbb{Q}(c) \geqslant 0$ for $-M \leqslant c \leqslant-1$ and in the first expression $\mathbb{Q}(c) \geqslant$ 0 for $c \leqslant-1$, with equality at $c=-1$, whereas in the second $\mathbb{Q}(c) \leqslant 0$ for $c<-M$ with equality at $c=-M$ and $c=-1$.

The absolute maximum of $\mathcal{Q}(c)$, over $(-\infty,-1]$, in (2.14) is at $c=-(2+\sqrt{3}) / 3$ with $Q^{\prime}(c)<0$ for $c \in(-(2+\sqrt{3}) / 3,-1]$.

In (2.15) the absolute maximum of $\mathscr{Q}(c)$ is at $c=c_{0}$ with

$$
c_{0}=M(M-1)(M+1+\sqrt{M}) /\left(1-M^{3}\right) \in[-M,-1] \text {. }
$$

If $c_{0} \leqslant-2 M / 3\left(c_{0}>-2 M / 3\right)$, then the maximum of $\mathbb{Q}(c)$ over $[-M,-2 M / 3]$ is at $c=c_{0}(c=-2 M / 3)$. 
We are now prepared to solve our problem when $\mathcal{H}=\left\{h: h \in L_{\infty}((0, a))\right.$, $h(x) \geqslant 1$ a.e. $\}$. This is the case that $M=\infty$ as no fixed upper bound is given for elements of $\mathcal{H}$.

THEOREM 2.1. Let $M=\infty$. Then

$\max \left\{\int_{0}^{a} p_{H}(x) d x: H \in \mathcal{H}\right\}=\int_{0}^{a} p_{H_{0}}(x) d x=\max \left\{\int_{I_{H}} p_{H}(x) d x: H \in \mathcal{H}\right\}$

where $H_{0}(x)=(2+\sqrt{3}) / 2$ for $x \in[0, \alpha]$ and $H_{0}(x)=1$ for $x \in[\alpha, a]$ with

$$
\alpha=-27 c_{0}^{2} a\left(1+c_{0}\right) /\left(4-27 c_{0}^{2}\left(1+c_{0}\right)\right), \quad c_{0}=-(2+\sqrt{3}) / 3,
$$

and $\mathbb{Q}\left(c_{0}\right)$ is given by (2.14).

Proof. In this case we always have that $-3 c / 2<M$ for all $c \in$ $(-\infty,-1]$. Hence only (2.9) applies. Now for any $c$ the triangle determined by the lines in (2.9) bound $p_{H}(x)$ for $H \in \mathcal{H}_{c}$. Hence their area is bounded by $\mathbb{Q}(c)$ as given in (2.14). However, from the first equation in (2.12) and from (2.13) we see there is an $H \in \mathcal{H}_{c}$, such that the associated solution to (2.1) is given by (2.9) and the values specified in the statement of the theorem yield the maximum area of the triangle. Since $p_{H_{0}}(x) \geqslant 0, I_{H_{0}}=[a, b]$ and our results are complete.

We show that the area in (2.14) is larger than the area in (2.15) when both are evaluated at the same value of $c \in[-M,-1]$; i.e.

$$
\begin{aligned}
-2 a^{2}(1+c) /( & \left.4-27 c^{2}(1+c)\right) \\
& \geqslant-a^{2}(M+c)(1+c) / 2\left(M+c-(1+c) M^{3}\right) .
\end{aligned}
$$

This is equivalent to having

$$
f(Y) \equiv \frac{4}{27}-Y^{2}(1+Y) \geqslant 0
$$

for $Y \equiv c / M \in[-1,-1 / M]$. Now $f^{\prime}(Y)=-Y(2+3 Y)$ is zero at $Y=$ $-2 / 3, f^{\prime \prime}\left(-\frac{2}{3}\right)=2>0, f\left(-\frac{2}{3}\right)=0, f(-1)=\frac{4}{27}$, and $f(-1 / M)=4 / 27-$ $(M-1) / M^{3} \geqslant 0$ with equality if and only if $M=\frac{3}{2}$. Therefore (2.17) is verified.

Note that the above also says that $(2.14)$ is equal to $(2.15)$ when $c=$ $-2 M / 3$.

Corollary 2.1. If $M \geqslant(2+\sqrt{3}) / 2$, then the conclusion of Theorem 2.1 remains valid.

Proof. Because of our constraint on $M$ the maximum of (2.14) is achieved and by (2.17) this maximum is larger than the maximum of (2.15).

Now we consider the remaining values of $M$; i.e., $M \in(1,(2+\sqrt{3}) / 2)$. 
THEOREM 2.2. If $M \in[3 / 2,(2+\sqrt{3}) / 2)$ and if

$$
H_{0}(x)= \begin{cases}M, & x \in[0, \alpha), \\ 1, & x \in[\alpha, a],\end{cases}
$$

with $\alpha=27 a M^{2}(M-1) /\left(4+27 M^{2}(M-1)\right)$, then $p_{H_{0}}(x)$ solves the problem in (2.16) and $\int_{0}^{a} p_{H_{0}}(x) d x=\mathbb{Q}(-M)$ where $\mathcal{Q}(c)$ is given in (2.14).

If $M \in(1,3 / 2)$ and if

$$
H_{0}(x)= \begin{cases}3 c_{0} / 2, & x \in[0, \alpha), \\ 1, & x \in[\alpha, a],\end{cases}
$$

with $c_{0}=M(M-1)(M+1+\sqrt{M}) /\left(1-M^{3}\right)$ and $\alpha=a M^{3}\left(c_{0}-1\right) /(M$ $\left.+c_{0}-\left(1+c_{0}\right) M^{3}\right)$, then $p_{H_{0}}(x)$ solves the problem in $(2.16)$ and $\int_{0}^{a} p_{H_{0}}(x) d x$ $=\mathbb{Q}\left(c_{0}\right)$ where $\mathbb{Q}(c)$ is given in (2.15).

Proof. The first part follows as (2.14) is a decreasing function of $c$ over the range specified. The second part is a consequence of the fact that $c_{0}$ maximizes (2.15).

\section{REFERENCES}

1. Lord Rayleigh, Notes on the theory of lubrication, Philos. Mag. 35 (1918), 1-12.

2. C. B. Morrey, Jr., Multiple integrals in the calculus of variations, Springer-Verlag, New York, 1966.

3. Multiple integral problems in the calculus of variations and related topics, Univ. California Publ. Math., Berkeley, 1943, pp. 1-130.

Department of Mathematics, Moravian College, Bethlehem, Pennsylvania 18018

Engineering Mechanics Department, General Motors Research Laboratories, WARREN, MichigAN 48090

Center for the Application of Mathematics, Lehigh University, Bethlehiem, PennsylVANIA 18015 